\title{
EVALUACIÓN HIGIÉNICA DE RIESGOS FÍSICOS EN EL SECTOR MADERERO DEL ECUADOR
}

\author{
ROBERTO BARRAgán MONRROY* \\ https://orcid.org/0000-0003-4682-5529 \\ BetTy GonZALez OSORIO* \\ https://orcid.org/0000-0002-2851-2660 \\ Kimberly Capa Salazar* \\ https://orcid.org/0000-0001-5232-3651 \\ Universidad Técnica Estatal de Quevedo, Ecuador
}

Recibido: 5 de mayo del 2021 / Aprobado: 13 de julio del 2021

doi: https://doi.org/10.26439/ing.ind2021.n41.5539

RESUMEN: El objetivo de la presente investigación fue evaluar los riesgos físicos a los que está expuesto el personal que cumple labores en los procesos productivos e industriales correspondientes al sector maderero. Se identificó en las industrias dos áreas clave del desempeño del recurso humano para la evaluación higiénica: en las áreas de cepillado y de aserrío existen máquinas y herramientas que se encuentran en constante funcionamiento, cubriendo toda la jornada laboral. Los métodos investigativos considerados fueron de observación, campo, cuantitativo, entrevistas y metodologías disponibles por el Instituto Nacional de Seguridad e Higiene en el Trabajo de España, complementadas con guías técnicas de ruido, calculando el nivel de exposición diario equivalente, incertidumbre y atenuación; de iluminación, realizando las mediciones en ocho puntos clave tomando en cuenta los valores legales en función del sector industrial; y de vibraciones, calculando los valores de aceleración y exposición diaria. Los niveles de riesgo encontrados en las evaluaciones cumplen con los límites legales establecidos en las normativas vigentes a excepción de las vibraciones, que afectan al sistema manobrazo y representan situaciones de riesgo considerables para los trabajadores.

PALABRAS CLAVE: ruido / iluminación / vibraciones / sector maderero / ergonomía / factores de riesgo

\footnotetext{
*Correos electrónicos: robertobarraganm81@gmail.com; bgonzalez@uteq.edu.ec; kimberly.kiki@hotmail.com
} 


\section{HYGIENIC EVALUATION OF PHYSICAL RISKS IN THE TIMBER SECTOR IN ECUADOR}

ABSTRACT: The objective of this research was to evaluate the physical risks to which personnel working in the production and industrial processes of the timber sector are exposed. Two critical areas of human resource performance were identified in the industries for the hygienic evaluation. In these areas, corresponding to planning and sawing, machines and tools that are in constant operation, covering the entire workday. The research methods considered were observation, field, quantitative, interviews, and methodologies available from the National Institute of Safety and Hygiene at Work of Spain, complemented by technical noise guides calculating the equivalent daily exposure level, uncertainty, and attenuation. Lighting was analyzed by taking measurements at eight key points taking into account the legal values according to the industrial sector, and vibrations by calculating acceleration and daily exposure values. The risk levels found in the evaluations comply with the legal limits established in the regulations in force, except for vibrations, affecting the hand-arm system and representing considerable risk situations for workers.

KEYWORDS: noise / lighting / vibrations / timber sector / ergonomics / risk factors 


\section{INTRODUCCIÓN}

A pesar de que en la Constitución del Ecuador y en el decreto 2393 existen artículos que protegen la salud del recurso humano, los trabajadores están expuestos a factores de riesgo ocupacionales durante el desempeño de sus labores (Capa et al., 2018). En décadas pasadas, la prevención de riesgos laborales era conocida como una cuestión ética, debido a que el empleador no tenía la obligación de proteger a sus trabajadores con el desarrollo normativo en materia de seguridad y salud en el trabajo; a la cuestión ética se le sumaba la obligación legal, aspecto importante en el desempeño de los trabajadores y los posibles riesgos a los que estaban expuestos (Saldaña et al., 2015).

Según el subregistro que cuenta el Instituto Ecuatoriano de Seguridad Social (IESS), en el Ecuador ocurren 80000 accidentes de trabajo al año y 60000 enfermedades profesionales como hipoacusia, pérdida de capacidad visual, de olfato, afectaciones músculo-esqueléticas y factores de riesgo psicosociales. Mientras más tarde se detecta o trata una enfermedad laboral, existe mayor probabilidad de generar una incapacidad (Martínez, 2016).

En el sector de la madera, a diario los trabajadores se exponen a riesgos muy diversos como es el caso de los higiénicos, algunos potencialmente severos que pueden originar accidentes laborales de carácter grave o enfermedades profesionales (Díaz, 2014). Como riesgo ocupacional, el ruido es reconocido desde la antigüedad; la exposición a este riesgo ocupacional es un problema de salud importante: la Organización Mundial de la Salud (OMS) calcula que 1100 millones de jóvenes de todo el mundo podrían estar en riesgo de sufrir pérdida de audición debido a prácticas auditivas perjudiciales. Más de 43 millones de personas de entre 12 y 35 años padecen una pérdida auditiva discapacitante debida a diferentes causas, lo que afecta las habilidades de comunicación y adecuación social y puede ocasionar daños al sistema auditivo que además alteran otros sistemas y disminuyen el aprovechamiento de la labor del trabajador (Picard et al., 2008; Sierra y Bedoya, 2016).

Por otro lado, la iluminación es un factor fundamental en los puestos de trabajo y áreas en general, debido a su relación directa con la salud visual en relación con varios aspectos, como los físicos y los ergonómicos, ligados a una iluminación óptima de acuerdo con las características de los puestos y áreas de trabajo; varios estudios plantean que la mala iluminación de un lugar aumenta las probabilidades de que los trabajadores cometan errores cuando se encuentran trabajando, lo que posibilita que ocurran accidentes por esta causa (Beltrán y Merchán, 2013), lo que empeora si en el área se emplean máquinas comunes del sector maderero como cepilladoras, sierras circulares, entre otras, y exista deficiencia o exceso de iluminación, pues las probabilidades que ocurra un accidente es elevada. 
Según Arias y Martínez (2016), desde un enfoque de seguridad y salud ocupacional, las exposiciones a vibraciones críticas en el sistema mano-brazo o en el cuerpo entero, ligadas a factores como el tiempo de exposición pueden provocar afectaciones considerables a la salud y al bienestar del personal laboral.

En los últimos años, el sector maderero ha adquirido mayor relevancia en la oferta exportadora del Ecuador, uno de los principales proveedores de madera de balsa en el mundo. Dicho sector ha sido uno de los que mayor crecimiento ha tenido, para el año 2016 alcanzó una participación del 0,36 \% con respecto al PIB. En la actualidad, Ecuador posee más de 20000 hectáreas entre bosques naturales y reforestados, siendo las zonas de mayor producción la provincia del Guayas, El Oro, Los Ríos y Pichincha (Corporación Financiera Nacional, 2017; Gonzalez et al., 2010).

Para lograr las exportaciones de balsa, las empresas necesitan contar con diversas áreas, donde los trabajadores puedan cumplir sus respectivas labores acorde a sus puestos de trabajo, en los mismos que se pueden presentar riesgos relacionados con la higiene industrial que pueden afectar negativa e irreversiblemente su salud si no se tiene un debido control de los mismos. Por este motivo se tomó en consideración para la evaluación de ruido, iluminación y vibraciones los parámetros establecidos en las guías técnicas disponibles por el Instituto Nacional de Seguridad e Higiene en el Trabajo de España, normas UNE-EN reguladas por la Asociación Española de Normalización y Certificación y límites máximos permisibles establecidos en el R. D. 286/2003, la Norma DIN 5035, y el R. D. 1311/2005, respectivamente, por cada riesgo.

De acuerdo con la bibliografía consultada, no se encontraron estudios similares a esta investigación, por lo tanto, la investigación se planteó como objetivo evaluar los riesgos físicos a los que está expuesto el personal que cumple labores en los procesos productivos e industriales correspondientes al sector maderero.

\section{MATERIALES Y MÉTODOS}

\section{1 Área de estudio}

La provincia Los Ríos se encuentra ubicada en el litoral ecuatoriano. Es parte vital de la cuenca del río Guayas, con una extensión de $7509 \mathrm{~km}^{2}$, equivalente al 2,8 \% del territorio nacional. Tiene una población de 778115 habitantes (Gonzalez et al., 2020). Se realizaron salidas de reconocimiento a las principales empresas madereras, y se utilizaron métodos de observación, de campo, cuantitativos y entrevistas; se identificaron dos empresas de mayor extensión, de las cuales se tomó en consideración dos áreas claves para la evaluación higiénica: la de aserrío y la de cepillado, debido a la presencia de máquinas en constante funcionamiento en toda la jornada laboral. 


\subsection{Ruido}

Para la evaluación higiénica de ruido se tuvo en consideración la cercanía de la fuente emisora de ruido; se distinguió así la fuente por puestos de trabajo y se realizó la evaluación por el tipo de exposición directa al ruido de los operadores, recibidores de madera y ayudantes de operadores y recibidores (seis puestos) que hacen referencia a un grupo homogéneo de exposición (GHE) en ambas áreas.

Para el número de mediciones y cálculo se tomó como referencia la norma UNE-EN ISO 912, basada en la jornada completa, debido a las características de los puestos de trabajo, y se realizaron tres mediciones en tres días hábiles diferentes, cubriendo toda la jornada, que en este caso es de seis horas. Si los resultados difieren en $3 \mathrm{~dB}$ o más, se debe realizar una medición adicional en la jornada laboral. Para este tipo de medición se utilizó un dosímetro TES-1354 en base al anexo III del R. D. 286/2006 de instrumentos de medición y condiciones de aplicación, previamente calibrado.

Para los cálculos de los valores medidos en el dosímetro se emplean las siguientes ecuaciones:

- Nivel de exposición diario equivalente $\left(\mathrm{L}_{\text {Aeq,d }}\right)$, que proporciona información sobre el nivel de exposición al ruido del personal laboral.

$$
L_{A e q, d}=L_{A e q, T}+10 \log \left[\frac{T}{8}\right]
$$

Donde:

T = Duración diaria de la exposición (horas).

$\mathrm{L}_{\text {Aeq, } \mathrm{T}}=$ Nivel de presión sonora equivalente en el período de tiempo $\mathrm{T}(\mathrm{dBA})$. Se calcula con la siguiente ecuación:

$$
L_{A e q, T}=10 \log \left[\frac{1}{N} \sum_{n=1}^{N} 10^{L_{A e q, T, n / 10}}\right]
$$

- Incertidumbre

Para un análisis más profundo, se calculó la incertidumbre basada en la función o jornada completa, tal como lo describen la guía técnica de ruido 2006 y la NTP 950:

$U^{2}=\sum_{m}\left\{C_{a, m}^{2}\left(u_{1, a m}^{2}+u_{2, m}^{2}+u_{3, m}^{2}+\left[\frac{4,34 C_{a, m}}{T m}\right]^{2} u_{1 b, m}^{2}\right\}\right.$

Donde:

$U_{1 a, m}=$ Es la incertidumbre estándar debida al muestreo de operación. 
$U_{1 b, m}=$ Es la incertidumbre estándar debida a la duración de la operación $m$.

$U_{2, m}=$ Es la incertidumbre estándar debido al instrumento de medición usado para la operación $m$.

$\mathrm{U}_{3, \mathrm{~m}}=$ Es la incertidumbre estándar debido a la imperfecta selección de la posición del micrófono en la operación $m$.

$\mathrm{T}_{\mathrm{m}}=$ Es el valor medio de los valores obtenidos del tiempo de duración de la operación $m$.

$\mathrm{C}_{\mathrm{a}, \mathrm{m}}=$ Es el coeficiente de sensibilidad correspondiente a la operación $\mathrm{m}$.

- Atenuación proporcionada por el uso actual de protectores auditivos

En base a las características de los protectores auditivos que utilizan los trabajadores, tapones 3M 1270 y orejeras 3M H510P3, se procedió a realizar el cálculo de atenuación acústica tal como lo describe la norma UNE-EN 458:2016, empleando el método de SNR y H, M y L:

Método del SNR

$$
L_{A}^{\prime}=L_{C}-S N R
$$

Donde:

$\mathrm{L}_{\mathrm{C}}=$ Niveles de presión sonora ponderado $(C)$.

SNR= Parámetro que disponen de fábricas los protectores auditivos (fichas técnicas).

Método $\mathrm{H}, \mathrm{M}$ y L

Para dicho cálculo se toma en consideración la diferencia entre $L_{C}$ y $L_{A}$ de la siguiente forma: si la diferencia $L_{C}-L_{A} \leq 2 \mathrm{~dB}$ se utiliza la primera ecuación; en caso de $L_{C}-L_{A} \geq 2 d B$ se utiliza la segunda ecuación:

$$
\begin{aligned}
& P N R=M-\frac{H-M}{4}\left[L_{C}-L_{A}-2\right] \\
& P N R=M-\frac{H-M}{8}\left[L_{C}-L_{A}-2\right]
\end{aligned}
$$

Donde:

$H, M$ y L= Parámetros que disponen de fábrica los protectores auditivos (fichas técnicas). 
$\mathrm{L}_{\mathrm{c}}=$ Niveles de presión sonora ponderado $(C)$.

$\mathrm{L}_{\mathrm{A}}=$ Niveles de presión sonora ponderado $(A)$ correspondiente al intervalo más desfavorable.

Para evidenciar si los protectores brindan una protección óptima, se toman en consideración los valores descritos en la norma UNE-EN 458:2016.

Con el valor de PNR se procedió a calcular el nivel de presión sonora efectivo ponderado (A) con la siguiente ecuación:

$$
L_{A}^{\prime}=L_{A}-P N R
$$

\subsection{Iluminación}

Para la evaluación higiénica de la iluminación se tomó en consideración la metodología descrita en la guía técnica de iluminación en conjunto con el R. D. 486/1997, del 14 de abril.

No obstante, para mantener un control efectivo, la evaluación de iluminación tendrá en cuenta valores legales en función del sector industrial, debido a que la medición se llevó a cabo en dos áreas de aserrío y de cepillado pertenecientes a empresas del sector maderero dedicadas a la exportación de madera, donde la dificultad de la percepción visual del personal laboral depende en gran parte de los niveles de iluminación de las tareas en el ámbito industrial. En la tabla 1 se describen cuatro niveles de iluminación correspondientes a cuatro tareas de actividad en base a la Norma DIN 5035, referente a niveles de iluminación recomendada en actividades industriales.

\section{Tabla 1}

Niveles de iluminación recomendadas en actividades industriales

\begin{tabular}{ccc}
\hline Tareas & Descripción & Iluminación (lux) \\
\hline Muy finas & $\begin{array}{r}\text { Observación constante y por mucho tiempo } \\
\text { de detalles al límite del poder visual. } \\
\text { Recintos y trabajos no incluidos en las } \\
\text { categorías I, III o IV. }\end{array}$ & 1000 \\
Normales & Se excluye la percepción de detalles pequeños. & $500-1000$ \\
Bastas & No se trabaja continuamente. & $250-500$ \\
\hline
\end{tabular}

Elaboración propia a partir de la norma DIN 5035

Para la medición de la iluminación se tomó en consideración toda el área de aserrío y cepillado, identificando cuatro puntos de mediciones clave cercanos a los tres puestos 
de trabajo de cada área; posteriormente se utilizó un luxómetro PM6612 previamente calibrado para la medición, los valores obtenidos se promediaron por el número de medición por cada área.

\subsection{Vibraciones}

Para la evaluación higiénica de vibraciones se tomó en consideración la metodología descrita en la guía para la evaluación y prevención de los riesgos relacionados con las vibraciones mecánicas en conjunto con la norma UNE-EN ISO 5349. Se identificó el empleo de maquinaria y herramientas generadoras de vibraciones por cada puesto de trabajo; los operadores y recibidores de ambas áreas están expuestos de forma directa a las vibraciones debido a su contacto directo con objetos vibrantes que afectan al sistema mano-brazo, a diferencia de los ayudantes que no requieren evaluación ya que no tienen contacto con ningún objeto que genere vibración. Para este tipo de medición se utilizó un vibrómetro PCE-VM 31 previamente calibrado de acuerdo con la norma UNE-EN ISO 8041.

Para los cálculos de la aceleración obtenida en el vibrómetro se utilizó las siguientes ecuaciones acorde a lo que establece la norma UNE-EN ISO 5349:

$$
a_{h v}=\sqrt{\left(a_{h w x}^{2}+a_{h w y}^{2}+a_{h w z}^{2}\right)}
$$

Donde:

$\left(a^{2}{ }_{h w x}+a^{2}{ }_{h w y}+a^{2}{ }_{h w z}\right)=$ Suma de los cuadrados de los componentes de la aceleración ponderada en frecuencia en los tres ejes.

En base al tiempo de exposición, se evidenció en los puestos de trabajo de las áreas de aserrío y cepillado que el contacto con los objetos vibrantes es relativamente corto, por ello se tomó una serie de mediciones a diferentes horas del día, como mínimo de un minuto. El promedio de las magnitudes de las vibraciones de una serie de números de muestras vienen dadas por la siguiente ecuación:

$$
a_{h w}=\sqrt{\frac{1}{T} \sum_{j=1}^{N} a_{h w j}^{2}} t_{j}
$$

Donde:

$a_{h w j}=$ Es la magnitud de las vibraciones medidas para la muestra $j$.

$\mathrm{t}_{\mathrm{j}}=$ Es la duración de la medición de la muestra $j$. 
Conocidos los valores de aceleración y tiempo de exposición se calculó el valor de exposición diaria $(A)$ dentro de la jornada correspondiente a ocho horas con la siguiente ecuación:

$$
A(8)=a_{h w} \sqrt{\frac{T_{e x p}}{T_{0}}}
$$

\subsection{Análisis de datos}

Para determinar si existen diferencias estadísticamente significativas entre los puestos de trabajo y áreas evaluadas, se empleó análisis estadísticos con la ayuda del software Infostat, en función de las variables de estudio (categóricas y numéricas). Para identificar si los niveles de las mediciones representan riesgos para los trabajadores, se tomó como referencia los límites máximos permitidos para ruido, atenuación, iluminación y vibraciones de acuerdo con los reales decretos y normas técnicas españolas y alemanas: R.D.286/2003, Norma UNE-EN 458, Norma DIN 5035 y R.D.1311/2005, respectivamente.

\section{RESULTADOS Y DISCUSIÓN}

\subsection{Ruido}

Sobre la base de la evaluación higiénica de ruido, se pudo evidenciar que en todos los puestos de trabajo de las áreas de aserrío y de cepillado los niveles de ruido superan los valores límite de exposición $L A e q, d>87 \mathrm{~dB}(\mathrm{~A})$. Los mayores niveles encontrados corresponden al operador de sierra del área de aserrío, con $93,2 \pm 3 \mathrm{~dB}$, y el menor nivel corresponde al ayudante de operador y recibidor del área de aserrío, con $88,8 \pm 3 \mathrm{~dB}$, tal como se evidencia en la tabla 2. Por tal motivo, todo el personal laboral debe de contar con protectores auditivos de manera obligatoria, ya que estos niveles corresponden a riesgos intolerables con consecuencias críticas en el sistema auditivo. En estos casos, para identificar si los trabajadores son vulnerables al ruido evaluado, se tendrá en cuenta la atenuación que procuran los protectores auditivos individuales utilizados por el personal laboral, tal como lo establece el artículo 5.2 del R. D.286/2006. 
Tabla 2

Resultados de los niveles equivalentes diarios e incertidumbres de ruido

\begin{tabular}{|c|c|c|c|c|c|c|}
\hline Áreas & Puestos & GHE & LAeq,T dB(A) & LAeq, d dB(A) & Incertidumbre & Intervalo \\
\hline \multirow{3}{*}{ Aserrío } & $\begin{array}{l}\text { Operador } \\
\text { de sierra }\end{array}$ & Operador & 94,4 & 93,2 & 3 & 90,2 y 96,2 \\
\hline & $\begin{array}{l}\text { Recibidor } \\
\text { de madera }\end{array}$ & Recibidor & 94,1 & 92,9 & 3 & 89,9 y 95,9 \\
\hline & $\begin{array}{l}\text { Ayudante } \\
\text { de operador } \\
\text { y recibidor }\end{array}$ & Ayudante & 90,0 & 88,8 & 3 & 85,8 y 91,8 \\
\hline \multirow{3}{*}{ Cepillado } & $\begin{array}{c}\text { Operador } \\
\text { de máquina } \\
\text { de cepillado }\end{array}$ & Operador & 95,0 & 93,8 & 3 & 90,8 y 96,8 \\
\hline & $\begin{array}{c}\text { Recibidor } \\
\text { de la } \\
\text { máquina } \\
\text { de cepillado }\end{array}$ & Recibidor & 94,3 & 93,1 & 3 & 90,1 y 96,1 \\
\hline & $\begin{array}{l}\text { Ayudante } \\
\text { de operador } \\
\text { y recibidor }\end{array}$ & Ayudante & 90,3 & 89,1 & 3 & 86,1 y 92,1 \\
\hline
\end{tabular}

Elaboración propia

De acuerdo con la prueba de Kruskal Wallis, se demostró que no existen diferencias estadísticamente significativas $(H=4,57 ; p=0,067)$ en los niveles de ruido de los grupos homogéneos de exposición (operadores, recibidores y ayudantes) de las áreas de aserrío y de cepillado (figura 1).

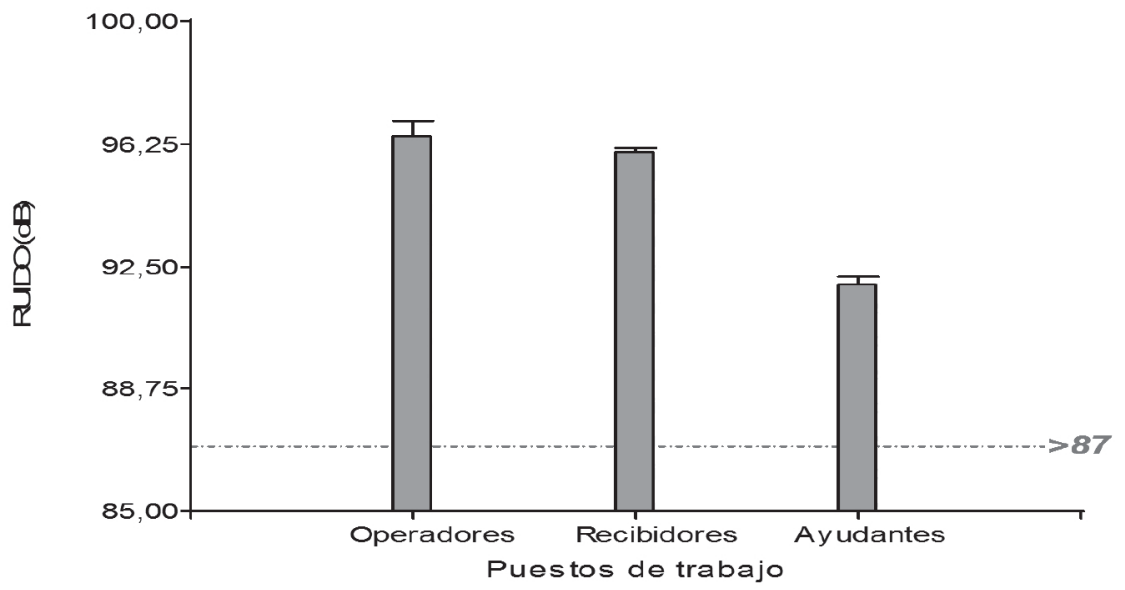

Figura 1. Resultados estadísticos de ruido

Elaboración propia 
Los dos métodos considerados para la evaluación de la atenuación proporcionada por los protectores auditivos arrojaron niveles similares, tal como se detalla la tabla 3 , que recoge la evaluación de la protección auditiva de los trabajadores obtenida por el método $\mathrm{H}, \mathrm{M}$ y L, por ser más exacto. Los resultados demuestran que los EPI que utiliza el personal laboral de ambas áreas brinda una protección satisfactoria y aceptable frente a los niveles de ruido que superan los $87 \mathrm{~dB}(\mathrm{~A})$. Los puestos de trabajo que muestran valores de exposición al ruido aceptables son los ayudantes, mientras que los operadores y recibidores muestran valores de exposición satisfactoria. Se trata de un aspecto positivo para la empresa y los trabajadores, pues muestra un nivel de riesgo trivial, categoría 1 con consecuencias tolerables.

Tabla 3

Atenuación proporcionada por el uso actual de protectores auditivos

\begin{tabular}{|c|c|c|c|c|c|c|c|}
\hline Áreas & Puestos & $\mathrm{LA} d \mathrm{~B}(\mathrm{~A})$ & $\mathrm{LC} d \mathrm{~B}(\mathrm{C})$ & PNR & $L^{\prime} A d B(A)$ & SNR & $L^{\prime} A d B(A)$ \\
\hline \multirow[t]{3}{*}{ Aserrío } & $\begin{array}{l}\text { Operador } \\
\text { de sierra }\end{array}$ & 96,2 & 99,4 & 21,7 & 74,5 & 26 & 73,4 \\
\hline & $\begin{array}{l}\text { Recibidor } \\
\text { de madera }\end{array}$ & 95,9 & 99,1 & 21,7 & 74,2 & 26 & 73,1 \\
\hline & $\begin{array}{c}\text { Ayudante } \\
\text { de operador } \\
\text { y recibidor }\end{array}$ & 91,8 & 93,9 & 21,9 & 69,9 & 25 & 68,9 \\
\hline \multirow[t]{3}{*}{ Cepillado } & $\begin{array}{c}\text { Operador } \\
\text { de máquina } \\
\text { de cepillado }\end{array}$ & 96,8 & 99,5 & 22,2 & 74,6 & 26 & 73,5 \\
\hline & $\begin{array}{c}\text { Recibidor } \\
\text { de la máquina } \\
\text { de cepillado }\end{array}$ & 96,1 & 99,1 & 21,9 & 74,2 & 26 & 73,1 \\
\hline & $\begin{array}{c}\text { Ayudante } \\
\text { de operador } \\
\text { y recibidor }\end{array}$ & 92,1 & 94,0 & 22,1 & 70,0 & 25 & 69 \\
\hline
\end{tabular}

Elaboración propia

La prueba de Kruskal Wallis demostró que no existen diferencias estadísticamente significativas $(H=4,57 ; p=0,067)$ en la atenuación proporcionada por el uso actual de protectores auditivos de los grupos homogéneos de exposición de las áreas de aserrío y de cepillado (figura 2). 


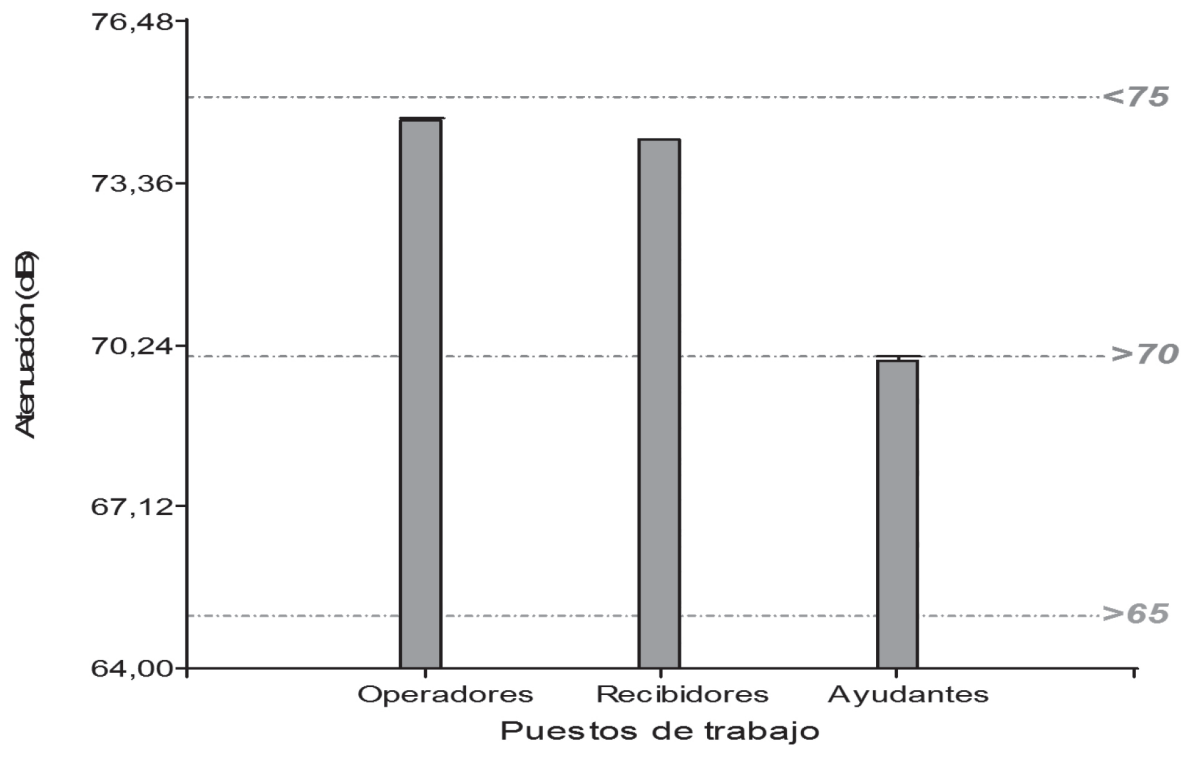

Figura 2. Resultados estadísticos de atenuación

Elaboración propia

\subsection{Iluminación}

Sobre la base de la evaluación higiénica, se pudo evidenciar que en las áreas de aserrío y de cepillado los niveles de iluminación se encuentran dentro de los valores legales de acuerdo con la actividad industrial, tipo de interior, tarea y actividad: 250-500 Lux. Los mayores niveles de iluminación encontrados pertenecen al área de aserrío, tal como se detalla en la tabla 4; se trata de un aspecto positivo para los trabajadores y la empresa, pues presentan un nivel de riesgo trivial, categoría 1 con consecuencias tolerables.

Tabla 4

Resultados de las mediciones de iluminación

\begin{tabular}{|c|c|c|c|c|c|}
\hline \multirow{2}{*}{$\begin{array}{c}\text { Áreas } \\
\text { Aserrío }\end{array}$} & \multicolumn{4}{|c|}{ Mediciones Em (Lux) } & \multirow{2}{*}{$\frac{\text { Em (Lux) }}{401,6}$} \\
\hline & 401,7 & 400,8 & 403,1 & 400,9 & \\
\hline Cepillado & 352,2 & 353,1 & 350,1 & 350,9 & 351,6 \\
\hline
\end{tabular}

Elaboración propia

De acuerdo con la prueba de Wilcoxon, se demostró que sí existen diferencias estadísticamente significativas ( $w=26,0 ; p=0,029)$ entre las dos áreas, siendo la media mayor en el área de aserrío $(401,63 \pm 1,06)$ que en la de cepillado $(351,58 \pm 1,34)$ en cuanto a los niveles de iluminación (figura 3). 


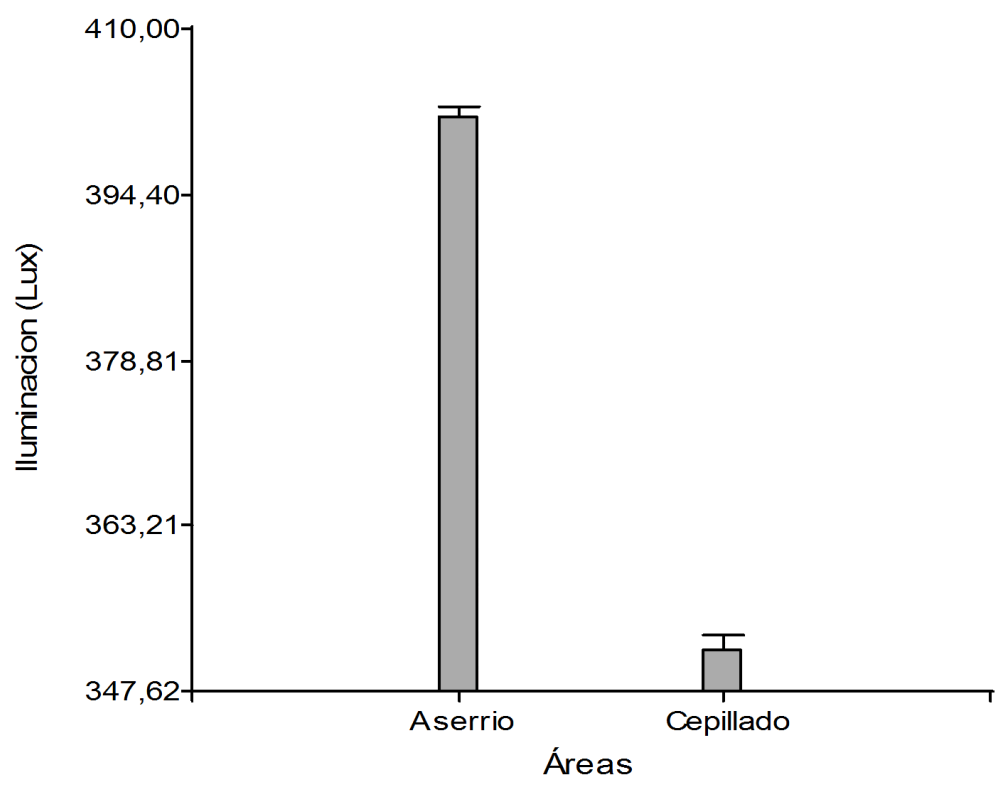

Figura 3. Resultados estadísticos de iluminación

Elaboración propia

\subsection{Vibraciones}

Sobre la base de la evaluación higiénica, se pudo evidenciar que todos los puestos de trabajo evaluados en las áreas de aserrío y de cepillado se encuentran en situación de riesgo, con valores de $A(8)<5 \mathrm{~m} / \mathrm{s} 2$ y A (8) $>2.5 \mathrm{~m} / \mathrm{s} 2$. Los mayores niveles de exposición diaria (A) a vibraciones, normalizados a lo largo de la jornada completa, corresponden al área de cepillado en el puesto de operador de la máquina de cepillado, tal como lo demuestra la tabla 5 . Se trata de un aspecto negativo para los trabajadores y la empresa, al presentar un nivel de riesgo moderado categoría III con consecuencias importantes. Por ello que es necesaria la implementación de planificaciones de la actividad preventiva, ya que estos niveles registrados podrían provocar afectaciones considerables al personal laboral. 
Tabla 5

Resultados de mediciones de vibraciones

\begin{tabular}{|c|c|c|c|c|c|c|}
\hline Áreas & Puestos & $A_{h v}$ & $\begin{array}{c}\text { Tiempo } \\
\text { de muestreo } \\
(\mathrm{Sg})\end{array}$ & $a_{h w}$ & $\begin{array}{c}\text { Jornada } \\
\text { (h) }\end{array}$ & $\mathrm{A}(8)\left(\mathrm{m} / \mathrm{s}^{2}\right)$ \\
\hline \multirow[t]{8}{*}{ Aserrío } & \multirow{4}{*}{$\begin{array}{c}\text { Operador de } \\
\text { cierra }\end{array}$} & 3,54 & 19 & \multirow[t]{4}{*}{3,51} & \multirow[t]{4}{*}{6} & \multirow[t]{4}{*}{3,04} \\
\hline & & 3,55 & 20 & & & \\
\hline & & 3,44 & 16 & & & \\
\hline & & 3,49 & 18 & & & \\
\hline & \multirow{4}{*}{$\begin{array}{c}\text { Recibidor de } \\
\text { madera }\end{array}$} & 3,37 & 19 & \multirow[t]{4}{*}{3,37} & \multirow[t]{4}{*}{6} & \multirow[t]{4}{*}{2,92} \\
\hline & & 3,42 & 16 & & & \\
\hline & & 3,30 & 20 & & & \\
\hline & & 3,38 & 19 & & & \\
\hline \multirow[t]{8}{*}{ Cepillado } & \multirow{4}{*}{$\begin{array}{l}\text { Operador de } \\
\text { la máquina } \\
\text { de cepillado }\end{array}$} & 3,60 & 17 & \multirow[t]{4}{*}{3,56} & \multirow[t]{4}{*}{6} & \multirow[t]{4}{*}{3,08} \\
\hline & & 3,55 & 15 & & & \\
\hline & & 3,52 & 16 & & & \\
\hline & & 3,55 & 19 & & & \\
\hline & \multirow{4}{*}{$\begin{array}{l}\text { Recibidor de } \\
\text { la máquina } \\
\text { de cepillado }\end{array}$} & 3,51 & 16 & \multirow[t]{4}{*}{3,51} & \multirow[t]{4}{*}{6} & \multirow[t]{4}{*}{3,04} \\
\hline & & 3,44 & 14 & & & \\
\hline & & 3,54 & 15 & & & \\
\hline & & 3,56 & 19 & & & \\
\hline
\end{tabular}

Elaboración propia

La prueba T student mostró que no existen diferencias estadísticamente significativas ( $T=1,26 ; p=0,333$ ) en los valores de exposición diaria (A) a vibraciones en los grupos homogéneos de exposición (figura 4).

Sobre la base de la evaluación higiénica del ruido se demostró que todos los puestos de trabajo de ambas áreas están expuestos a niveles de ruido que superan los valores límite de exposición $\mathrm{L}_{\text {aeq,d }}>87 \mathrm{~dB}(\mathrm{~A})$; sin embargo la protección proporcionada por el uso actual de protectores auditivos frente a este riesgo brinda una protección aceptable para los ayudantes $>65 \mathrm{~dB}$ y una protección satisfactoria para los operadores y recibidores $>70 \mathrm{~dB}<75 \mathrm{~dB}$. Estos resultados concuerdan con la investigación realizada por González (2016) en el Ecuador, donde los protectores auditivos cumplen el rol de proteger satisfactoriamente al personal laboral frente a niveles de ruido que superan las normas internacionales españolas y alemanas. 


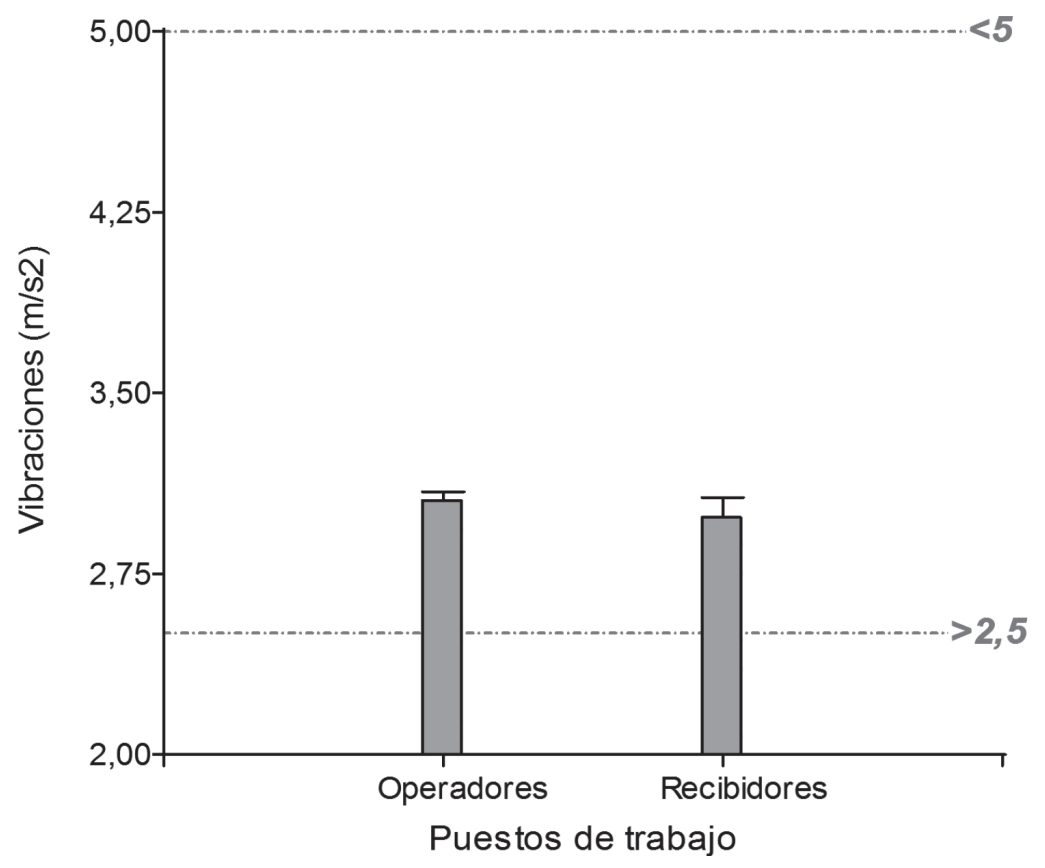

Figura 4. Resultados estadísticos de vibraciones

Elaboración propia

Los niveles de iluminación encontrados cumplen con los límites legales establecidos de 200-500 Lux, aspecto positivo para los trabajadores y las empresas, debido a su influencia directa en la salud visual. Por otra parte, los trabajadores de las áreas de aserrío y de cepillado están expuestos a situaciones de riesgo a vibraciones en el sistema mano-brazo que superan los límites legales $>2,5 \mathrm{~m} / \mathrm{s}^{2}<5 \mathrm{~m} / \mathrm{s} 2$, aspecto negativo para el personal laboral y la empresa. Estos niveles pueden provocar afectaciones significativas a largo plazo (Arias y Martínez, 2016).

\section{CONCLUSIONES}

Al tratarse de empresas pertenecientes al sector maderero, el personal laboral está expuesto a una diversidad de riesgos ligados con la higiene industrial, debido a la complejidad de sus procesos productivos en los que la presencia de herramientas y máquinas es constante. Si no existe un debido control o prevención de los niveles de estos riesgos, las afectaciones a la salud podrían tornarse irreversibles. 
Los niveles encontrados cumplen con los límites legales, con excepción de las vibraciones, lo que representa una situación de riesgo que puede provocar problemas vasculares, de huesos, entre otros, por lo que es necesario implementar medidas correctoras y preventivas que fortalezcan el diseño de estrategias que conduzcan a la prevención y cumplimiento legal articulados por parte de las empresas dedicadas a la fabricación de los derivados de la madera.

Los resultados de la evaluación higiénica de ruido demostraron que en todos los puestos de trabajo de las áreas de aserrío y de cepillado se superan los valores límites de exposición; sin embargo, la atenuación proporcionada por el uso actual de protectores auditivos representa una protección aceptable y satisfactoria frente a este riesgo, lo que resulta positivo para el personal laboral y la empresa; ello no obstante, es recomendable llevar controles trianuales sobre los niveles de ruido generados en estos puestos.

Con respecto a los niveles de iluminación, se concluyó que en ambas áreas existen niveles lumínicos aceptables para el desarrollo normal de las actividades, lo que representa un aspecto positivo tanto para el personal laboral como para las empresas, ya que se cumple con la normativa vigente; se evita así que un lugar con poco o exceso de iluminación pueda representar un problema grave para los trabajadores, ya que las posibilidades de que ocurran afectaciones visuales o accidentes es alta. De igual manera, es recomendable llevar un control trianual frente a dicho riesgo.

La evaluación higiénica de riesgos físicos realizada en torno al sector maderero permitió conocer los niveles de ruido, iluminación y vibraciones a los que está expuesto el personal laboral de las áreas de aserrío y de cepillado, y permitió disponer de un referente apropiado de indicadores de riesgos físicos para sustentar el estudio y dar solución al problema identificado y, con ello, mejorar la seguridad y salud ocupacional del sector evaluado.

\section{REFERENCIAS}

Arias, G., y Martínez, C. (2016). Evaluación de la exposición al riesgo por vibraciones en el segmento mano brazo en compañías del sector metalmecánico. Medicina y Seguridad Del Trabajo, 62(245), 327-336. http://scielo.isciii.es/scielo. php?script=sci_arttext\&pid=S0465-546X2016000500005

Asociación Española de Normalización y Certificación. (2002). Medición y Evaluación de La Exposición Humana a Las Vibraciones Transmitidas Por La Mano, (UNE-EN ISO 5349-2). https://www.une.org/encuentra-tu-norma/busca-tu-norma/norma/?c $=\mathrm{N} 0056117$

Asociación Española de Normalización y Certificación. (2009). Determinación de la exposición al ruido en el trabajo, (UNE-EN ISO 9612). https://www.une.org/ encuentra-tu-norma/busca-tu-norma/norma?c=N0044443 
Asociación Española de Normalización y Certificación. (2016). Protectores auditivos. Recomendaciones relativas a la selección, uso, cuidado y mantenimiento, (UNE-EN 458:2016). https://www.une.org/encuentra-tu-norma/busca-tu-norma/norma?c= N0056361

Asociación Española de Normalización y Certificación. (2018). Respuesta humana a las vibraciones. Instrumentos de medida, UNE-EN ISO 8041-1. https://www.google. com/search?client=firefox-b-d\&sxsrf=ALeKk019cu8BoWAP7JNcjkQg-u3t J NK94g\%3A1608787567567\&ei=bybkX_aLIoiQ5wLc3LIw\&q=UNE$\mathrm{EN}+\mathrm{ISO}+8041+\& \mathrm{oq}=\mathrm{UNE}-\mathrm{EN}+\mathrm{ISO}+8041+\& \mathrm{gs}$ Icp $=\mathrm{CgZwc3ktYWIQAzIGCAAQFhA}$ eOgQIABBHUPWiAlj1ogJgp6oCaABwAngAgAGjAYgBowGSAQMwLjGYAQC

Beltrán, J., y Merchán, C. (2013). Niveles de iluminación y su relación con los posibles efectos visuales en los empleados de una IPS de Bogotá. Movimiento Científico, 7(1), 31-37. https://doi.org/10.33881/2011-7191.

Capa, L. B., Flores Mayorga, C., y Sarango Ortega, Y. (2018). Evaluación de factores de riesgos que ocasionan accidentes laborales en las empresas de MachalaEcuador. Universidad y Sociedad, 10(2), 335-340. http://scielo.sld.cu/pdf/rus/ v10n2/2218-3620-rus-10-02-341.pdf

Corporación Financiera Nacional. (2017). Explotación de viveros forestales y madera en pie. https://www.cfn.fin.ec/wp-content/uploads/2017/10/Ficha-Sectorial-Viverosy-Madera-en-Pie.pdf

Díaz, Á. (2014). Riesgos específicos y su prevención en el sector de la madera. En Manual básico de seguridad y salud en el trabajo. Invassat.

González, Barragan, R. M., Luis, S., y Marisol, R. (2020). Influencia de las variables climáticas en el rendimiento de cultivos transitorios en la provincia Los Ríos, Ecuador. Centro Agrícola, 47(4), 54-64. http://cagricola.uclv.edu.cu/descargas/ pdf/V47-Numero_4/cag07420.pdf

González, Cervantes, X., Torres, E., Sánchez, C., y Simba, L. (2010). Caracterización del cultivo de balsa (Ochroma pyramidale) en la provincia de Los Ríos, Ecuador. Ciencia y Tecnología, 3(2), 7-11. http://www.erevistas.csic.es/ficha_articulo. php?url=oai_revista718:65\&oai_iden=oai_revista718

González, J. (2016). Evaluación higiénica de ruido y vibraciones en los Viveros Municipales Tejavana (Córdoba) [Tesis de posgrado, UNIR]. https://reunir.unir.net/handle/ $123456789 / 4535$

Instituto Alemán de Normalización. (2006). Iluminación artificial. Parte 6: Medición y evaluación, (DIN 5035-6:2006-11). https://www.aenor.com/normas-y-libros/ buscador-de-normas/din/?c=90757408 
Instituto Nacional de Seguridad e Higiene en el Trabajo. (2012). NTP 950: Estrategias de medición y valoración de la exposición a ruido (I): Incertidumbre de la Medición. http://www.insht.es/InshtWeb/Contenidos/Documentacion/NTP/NTP/ Ficheros/926a937/950w.pdf

Instituto Nacional de Seguridad e Higiene en el Trabajo. (2005). Guía Técnica Para La Evaluación y Prevención de Los Riesgos Relacionados Con Las Vibraciones Mecanicas. https://www.insst.es/documents/94886/203536/Guía+Técnica+par a+la+evaluación+y+prevención+de+los+riesgos+relacionados+con+las+vibraci ones+mecánicas/817c3e60-3256-4bdb-accb-e25b430cd91e

Instituto Nacional de Seguridad e Higiene en el Trabajo. (2006). Guía Para La Evaluación y Prevención de Los Riesgos Relacionados Con La Exposición de Los Trabajadores Al Ruido. https://www.insst.es/documents/94886/96076/guía_técnica_ruido. pdf/85821846-2195-4359-94eb-08fdc6457dce

Instituto Nacional de Seguridad e Higiene en el Trabajo. (1989). Iluminación en el puesto de trabajo. https://www.adpreven.es/wp-content/uploads/2016/02/lluminacionen-el-puesto-de-trabajo.pdf

Martínez Guirao, J. E. (2016). Riesgos laborales en la construcción. un análisis sociocultural. Universitas, 23, 65. https://doi.org/10.17163/uni.n23.2015.03

Organización Mundial de la Salud. (2015). Departamento de Enfermedades No Transmisibles, Discapacidad y Prevención de La Violencia y Los Traumatismos (NVI). http://www.who.int/pbd/deafness/activities/MLS_Brochure_Spanish_lowres _for_web.pdf

Picard, M., Girard, S. A., Courteau, M., Leroux, T., Larocque, R., Turcotte, F., Lavoie, M., y Simard, M. (2008). Could driving safety be compromised by noise exposure at work and noise-induced hearing loss? Traffic Injury Prevention, 9(5), 489-499. https://doi.org/10.1080/15389580802271478

Real Decreto 286/2006 de 10 de marzo, sobre la protección de la salud y la seguridad de los trabajadores contra los riesgos relacionados con la exposición al ruido. Boletín Oficial Del Estado, núm. 60, 1-12. http://www.insht.es/InshtWeb/ Contenidos/Normativa/TextosLegales/RD/2006/286_2006/PDFs/realdecreto2862006de10demarzosobrelaprotecciondelasal.pdf

Real Decreto 1311/2005, de 4 de noviembre, sobre la protección de la salud y la seguridad de lostrabajadores frente a los riesgos derivados oque puedan derivarse dela exposición a vibraciones mecánicas. Boletín Oficial Del Estado, núm. 265, 1-11. http://www. insht.es/InshtWeb/Contenidos/Normativa/TextosLegales/RD/1999/1254_99/ PDFs/realdecreto12541999de16dejulioporelqueseapruebanlasmTxtAnt.pdf 
Saldaña, A., Hernando, L., y Núñez, J. (2015). Fundamentos de las técnicas de mejora de las condiciones de trabajo y ámbito jurídico de la prevención. Logroño - La Rioja: Universidad Internacional de La Rioja (UNIR). Material No Publicado.

Sierra, C., y Bedoya, M. (2016). Prevalence of Sensorineural Hearing Loss Induced Noise in Wood Sector Companies City Cartagena. Nova, 14(25), 47-56. http://www.scielo. org.co/scielo.php?script=sci_arttext\&pid=S1794-24702016000100005\&lng=en\& $\mathrm{nrm}=\mathrm{iso} \& \operatorname{tng}=$ 
